\title{
Enzymhistochemische Untersuchungen der Adenosin-Triphosphatase, der alkalischen und sauren Phosphatase sowie der Succinodehydrogenase im Leberparenchym der Ratte während der Einwirkung des Cancerogens Diäthylnitrosamin
}

\author{
P. BöRNER und W. GösSNer \\ Abteilung für physikalische Biologie des Max-Planck-Instituts für Virusforschung, Tübingen \\ und Pathologisches Institut der Universität Tübingen* \\ (Z. Naturforschg. 23 b, 1085-1091 [1968] ; eingegangen am 23. Dezember 1967)
}

\begin{abstract}
Der Einfluß von Diäthylnitrosamin in cancerogenen Dosen auf die Adenosin-Triphosphatase, alkalische und saure Phosphatase sowie die Succinodehydrogenase der Rattenleberzelle wird mit enzymhistochemischen Methoden untersucht. Diese Enzyme wurden ausgewählt, da sie an charakteristische Strukturen der Leberzelle gebunden sind.

DÄNA führt zu folgenden enzymhistochemischen Veränderungen im Leberparenchym: Schwund der ATPase aus umschriebenen Bereichen des Parenchyms. Aufhebung der peribiliären Anordnung der durch ihren Gehalt an saurer Phosphatase dargestellten Lysosomen. Diffuse Verteilung der Lysosomen im Cytoplasma der Leberzelle. In späteren Phasen der Cancerogenese wird die peribiliäre Anordnung der Lysosomen teilweise wiederhergestellt. Allmähliche Herabsetzung der Aktivität des Mitochondrienenzymes Succinodehydrogenase in ausgedehnten Parenchymbezirken. Auftreten von alkalischer Phosphatase in der Wand einzelner Leberzellen in späten Phasen der Cancerogenese.

Die Bereiche des Leberparenchyms, in denen es zum Schwund der ATPase der Gallenkapillaren und zu Veränderungen in der Anordnung der Lysosomen kommt, weisen jeweils auch einen Verlust der Glucose-6-Phosphatase auf.
\end{abstract}

Histochemische Untersuchungen des Leberparenchyms der Ratte in sehr frühen Phasen der Cancerogenese haben sich bisher besonders mit dem Verlust von Komponenten des endoplasmatischen Reticulums der Leberzelle beschäftigt. So beobachtete WEILER ${ }^{1}$ mit immunohistochemischen Methoden den allmählichen Schwund des sog. gewebsspezifischen Antigens, beschrieben Gössner und Friedrich-Freksa ${ }^{2}$ den Verlust der Glucose-6-Phosphatase in Gruppen von Leberzellen als zur Zeit früheste mit morphologischen Methoden nachweisbare Veränderungen im Ablauf der Cancerogenese. Sowohl das gewebsspezifische Antigen als auch die Glucose-6-Phosphatase sind Bestandteile des endoplasmatischen Reticulums der Leberzelle. In vorliegender Arbeit soll zum Vergleich auch das Verhalten einiger an andere charakteristische Strukturen der Leberzelle gebundener Enzyme unter dem Einfluß des Cancerogens Diäthylnitrosamin untersucht werden. Als Enzyme der Zellmembran wurden die Adenosin-Triphosphatase und die alkalische Phosphatase, als Lysosomenenzym die saure Phosphatase und aus der Gruppe der Mitochondrienenzyme die Succinodehydrogenase zur Untersuchung ausgewählt.

* Direktor des Instituts war bei Ausführung der Arbeit Prof. Dr. E. LetTerer.

1 E. Weiler, Z. Naturforschg. 11 b, 31 [1956].

\section{Methodik}

Im Rahmen eines anderen Versuches erhielten 34 Ratten vom Stamm Sprague-Dawley über einen Zeitraum von $4-20$ Wochen sechsmal wöchentlich $5 \mathrm{mg}$ Diäthylnitrosamin $/ \mathrm{kg}$ Körpergewicht mit der Schlundsonde verabreicht. Die Tiere wurden im Abstand von 1-2 Wochen getötet und ihre Leber enzymhistochemisch untersucht. Einzelheiten der Versuchsanordnung, der Fütterungsdauer und der den einzelnen Versuchsruppen verabreichten Gesamtdosen von Diäthylnitrosamin (DÄNA) s. bei GössNer, Friedrich-Freksa und Börner ${ }^{3}$.

Tötung der Tiere in Äthernarkose. Enzymhistochemischer Nachweis der Adenosin-Triphosphatase (ATPase), der alkalischen Phosphatase und der sauren Phosphatase an formalinfixierten Gefrierschnitten, die eine einwandfreie Darstellung der Enzymstrukturen gewährleisten. Nachweis der Succinodehydrogenase an unfixierten Kryostatschnitten. Daneben Vergleich der beobachteten enzymhistochemischen Veränderungen der ATPase, der alkalischen und sauren Phosphatase mit Glucose-6-Phosphatase-Verlusten im Leberparenchym an im Kryostaten angefertigten Serienschnitten. Diese sind wegen ihrer schlechteren Darstellung feiner Enzymstrukturen nur zum Vergleich der Lokalisation von enzymhistochemischen Veränderungen geeignet.

Nachweismethoden: Fixierung kleiner Gewebsstücke bei $+4{ }^{\circ} \mathrm{C} 20 \mathrm{Stdn}$. in 4-proz. Formalin mit Zusatz

2 W. Gössner u. H. Friedrich-Freksa, Z. Naturforschg. 19 b, 862 [1964].

${ }^{3}$ W. Gössner, H. Friedrich-Freksa u. P. Börner, Z. Krebsforschg., in Vorbereitung. 
von 1\% Calciumchlorid. Anschließend 24-stdg. Aufbewahrung in einer 30-proz. Rohrzuckerlösung mit Zusatz von $1 \%$ Gummi arabicum. Herstellung von Gefrierschnitten zum Nachweis folgender Enzyme:

Nachweis der Adenosin-Triphosphatase: Bleisalz-Methode nach Go mo ri, modifiziert nach Wachstein und Meisel ${ }^{4}$.

Nachweis der alkalischen Phosphatase: CalciumCobalt-Methode nach Gomori ${ }^{5}, \mathbf{7}$.

Nachweis der sauren Phosphatase: Bleisalz-Methode nach Gomori ${ }^{6,7}$.

Zur Herstellung unfixierter Gewebsschnitte Entnahme von Gewebsstückchen von etwa $0,5 \cdot 0,5 \mathrm{~cm}$ Grundfläche aus einem großen Leberlappen. Sofortiges Einfrieren durch ausströmende Kohlensäure. Anfertigung von Schnitten im Kryostat nach Dit tes-Duspiva bei $-20{ }^{\circ}$ C. Schnittdicke $10 \mu$. Antauen der Schnitte auf Deckgläschen. Nach kurzer Lufttrocknung wurden folgende Reaktionen durchgeführt:

Nachweis der Succinodehydrogenase: Tetrazolium-Salz-Methode nach Seligm a n und Rutenburg ${ }^{8}$, modifiziert nach Wat ten b e r g ${ }^{8 \mathrm{a}}$.

Nachweis der Glucose-6-Phosphatase nach Wachstein und Meisel ${ }^{9}$.

\section{Ergebnisse}

\section{Veränderungen im enzymhistochemischen Bild der Adenosin-Triphosphatase}

Die von uns angewandte ATPase-Darstellung zeigt eine hohe Aktivität des Enzyms in der Wand der interzellulären Gallenkapillaren. Weniger regelmäßig und schwächer ist ATPase auch in den Sinuswandungen nachweisbar. Ebenfalls positiv reagieren die Wände von Gefäßen und Gallengängen sowie das Bindegewebe in Periportalfeldern. Die in den Mitochondrien lokalisierte ATPase wird nicht dargestellt.

Mit dem nach 7-wöchiger DÄNA-Fütterung einsetzenden Verlust der Glucose-6-Phosphatase in Gruppen von Leberzellen geht eine starke Verminderung der ATPase-Aktivität im gleichen inselförmigen Gewebsbezirk einher. Ein großer Teil des dichten Netzwerkes der interzellulären Gallenkapillaren ist durch den Schwund der ATPase nicht mehr darstellbar. Andere Abschnitte des Kapillarsystems zeigen

4 M. W Achstein and E. Meisel, Amer. J. clin. Pathol. 27, 13 [1957].

5 G. Gomori, J. cellular comparat. Physiol. 17, 71 [1941 b].

6 G. Gomori, Arch. Pathology 41, 121 [1941].

7 G. Gomori, Microscopic Histochemistry, University of Chicago Press 1952.

7 A. M. Seligman and A. M. Rutenburg, Science [Washington] 113, 317 [1951]. eine herabgesetzte Enzymaktivität und sind im enzymhistochemischen Bild nur schattenhaft sichtbar. In den Sinuswänden findet sich weiterhin stellenweise eine schwache Enzymreaktion (Abb. 1 a).

Die späteren Phasen der Cancerogenese führen zu einer Ausbreitung und Intensivierung der inselförmigen ATPase-Verluste. In den Bereichen des Enzymschwundes treten Abschnitte der Gallenkapillaren und der Sinuswandungen - wenn überhaupt immer schattenhafter hervor (Abbn. $1 \mathrm{~b}$ und c). ATPase-Aktivität kann in den hepatocellulären Carcinomen völlig fehlen.

\section{Veränderungen im enzymhistochemischen Bild der alkalischen Phosphatase}

Im Lebergewebe der gesunden Ratte ist alkalische Phophatase nur bisweilen in der Wand größerer Gallengänge, in Gefäßendothelien und im periportalen Bindegewebe nachweisbar. Außerdem geben vereinzelt Leukozyten in den Sinusoiden eine positive Enzymreaktion (Abb. 2 a). Eine Veränderung dieser Befunde tritt erst in den Endstadien der Cancerogenese ein. Nach 20-wöchiger Fütterung von DÄNA läßt sich alkalische Phosphatase in der Wand von einzelnen Leberzellen oder Zellgruppen nachweisen (Abb. 2 b). Hepatocelluläre Carcinome zeigen eine sehr unterschiedliche Enzymaktivität. Neben Tumoren mit starkem Gehalt an alkalischer Phosphatase in der Wand der Tumorzellen finden sich solche ohne jede Enzymaktivität (Abb. 2 c).

\section{Veränderungen im enzymhistochemischen Bild der sauren Phosphatase}

Im Cytoplasma der Rattenleberzelle läßt sich saure Phosphatase in scharf begrenzten, kleinen, runden Granula von intensiver Enzymaktivität nachweisen. Diese Granula entsprechen den Lysosomen (Essner und Novikoff $)^{10}$. Sie sind vorwiegend peribiliär, entlang den Gallenkapillaren angeordnet. Im übrigen Cytoplasma sind sie nur vereinzelt anzutreffen. Eine starke Enzymaktivität findet sich auch in Sternzellen.

Nach 7-wöchiger Fütterung von DÄNA kommt es in umschriebenen inselförmigen Parenchymbezirken

8a L. W. Wattenberg and J. L. Leong, J. Histochem. Cytochem. 8, 296 [1960].

9 M. W [Baltimore] 4, 592 [1956].

10 E. Essner and A. B. Novikoff, J. biophysic. biochem. Cytol. 9, 773 [1961]. 

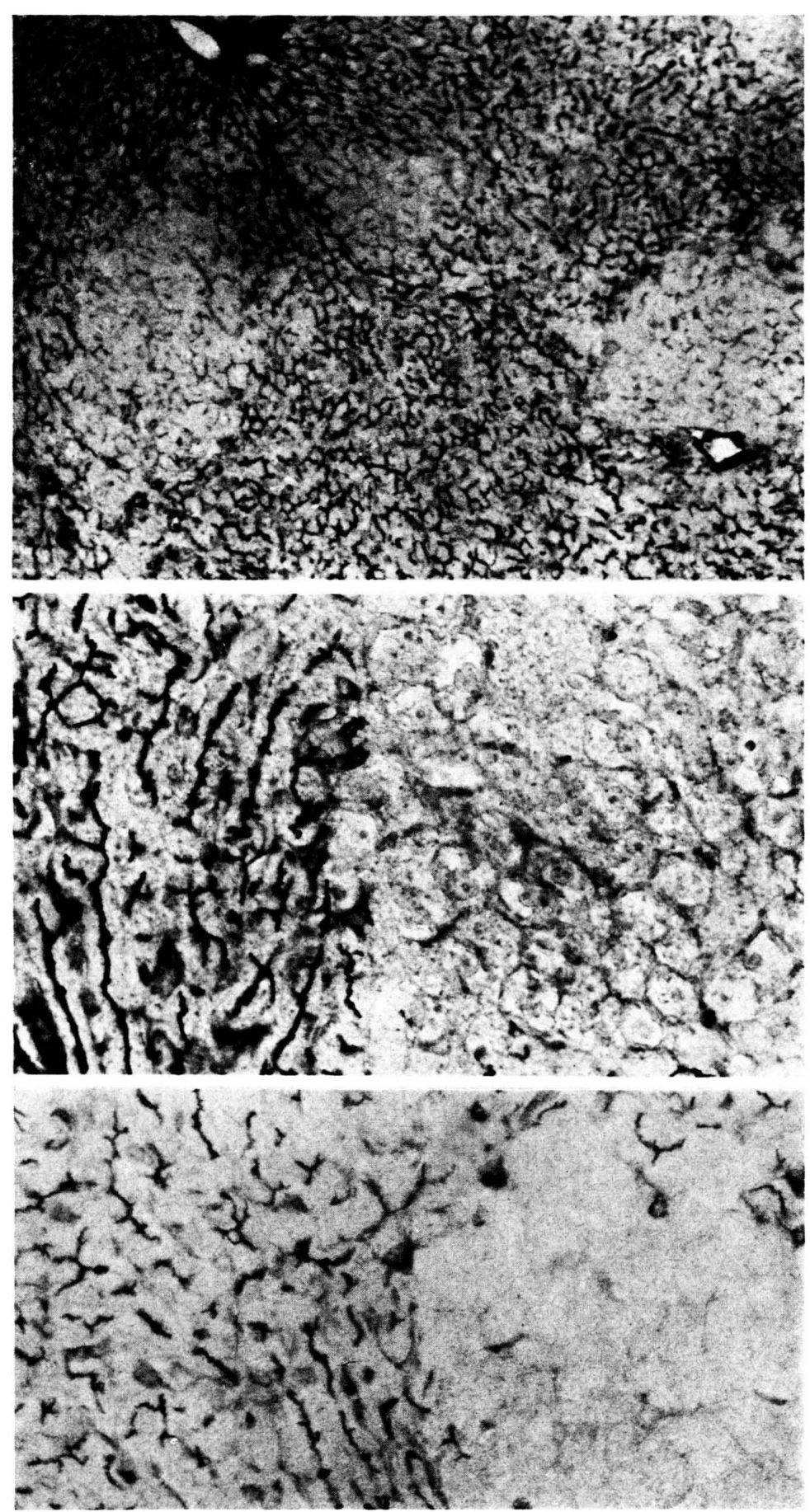

Abb. 1. Veränderungen der ATPase unter dem Einfluß von DÄNA. Zunehmender Schwund des Enzyms in umschriebenen Bezirken des Leberparenchyms. a: nach 8-wöchiger DÄNA-Fütterung, Vergr. 120-fach; b: nach 10-wöchiger DÄNA-Fütterung, Vergr. 750-fach; c: nach 14-wöchiger DÄNA-Fütterung, Vergr. 750-fach.

Abb. 1 a. im Zentrum einzelner Leberläppchen zu einer Auflösung der streng peribiliären Anordnung der enzymaktiven Granula. Ihre Anzahl erscheint vermindert. Die einzelnen Lysosomen sind nicht vergrößert. Sie sind jetzt diffus im Cytoplasma verteilt (Abb. 3 a). Lokalisation und Ausdehnung dieser enzymhistochemischen Veränderungen entsprechen jeweils einem Gewebsbereich, in dem die Leberzellen die 


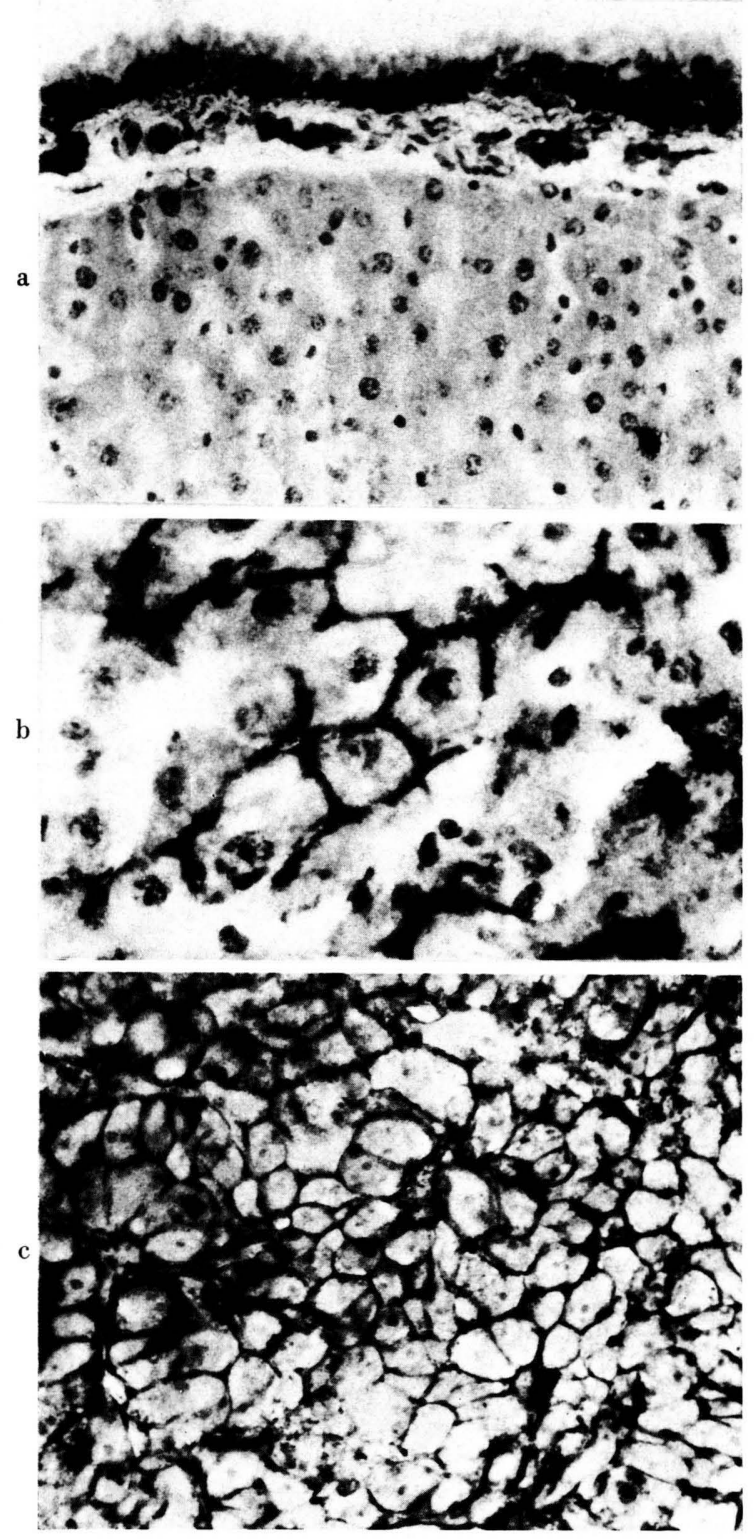

Abb. 2. Darstellung der alkalischen Phosphatase. a : normale Rattenleber. Die Zellwände sind frei von Enzym. Kernfärbung mit Hämalaun. Vergr. 625-fach. b: Auftreten alkalischer Phosphatase in der Wand einzelner Leberzellen nach 16-wöchiger Fütterung von DÄNA. Kernfärbung mit Hämalaun. Vergr. 1200-fach. c: Alkalische Phosphatase in den Zellwänden eines hepatocellulären Carcinoms. Vergr. 750-fach.

Glucose-6-Phosphatase-Aktivität verloren haben.

Nach 10-wöchiger Verabreichung von DÄNA verdrängt die starke Einlagerung von Glykogen in Glucose-6-Phosphatase freien Leberzellen das Cytoplasma oft zu einem schmalen Saum an die Zellwand. Bei Darstellung der sauren Phosphatase findet sich dieser Plasmasaum durch die dort zusammengeschobenen enzymaktiven Granula deutlich markiert. Das Innere des Zelleibes ist frei von Lysosomen (Abb. 3 b).

In den späten Stadien der Cancerogenese, nach 16-wöchiger Fütterung von DÄNA findet sich in Parenchymbezirken, die durch Vermehrung der Basophilie und Schwund des Glykogens im Cytoplasma gekennzeichnet sind, eine teilweise Wiederherstellung der peribiliären Anordnung der Lysosomen (Abb. 3 c). Die Anzahl enzymaktiver Sternzellen hat bis zu dieser Phase der Cancerogenese weder auffällig zu- noch abgenommen. In hepatocellulären Carcinomen stellt sich die saure Phosphatase in feineren und gröberen, unregelmäßig in der Zelle verteilten Granula dar.

\section{Veränderungen im enzymhistochemischen Bild der Succinodehydrogenase}

In der Leber der gesunden Ratte läßt sich die Succinodehydrogenase mit der von uns angewendeten Methode durch das Auftreten granulärer Formazanniederschläge im Cytoplasma nachweisen. Obwohl es sich bei der Succinodehydrogenase um ein nur in den Mitochondrien lokalisiertes Enzym handelt, dürfen diese Formazangranula nicht ohne weiteres als Darstellung einzelner Mitochondrien angesehen werden. Veränderungen des vom normalen Leberparenchym der Ratte bekannten enzymhistochemischen Bildes fanden sich erst nach 16-wöchiger Fütterung von DÄNA. Dann wird in ausgedehnten Parenchymbezirken der Leber der Gehalt der Zellen an Succinodehydrogenase vermindert. Die Formazanniederschläge in den Leberzellen sind geringer. Die einzelnen Formazangranula sind nicht vergröbert. Die unscharfe Begrenzung dieser Enzymverminderungen macht eine Zuordnung zu Glucose-6-Phosphatase freien Gewebsbezirken schwierig.

\section{Diskussion}

Im Gegensatz zu den am endoplasmatischen Reticulum nachweisbaren histochemischen Veränderungen können die hier beschriebenen Veränderungen der an andere charakteristische Strukturen der Leberzelle gebundenen Enzyme nicht als für die Cancerogenese spezifisch angesehen werden. Gleiche Befunde lassen sich bei Einwirkung der verschiedensten Noxen auf das Leberparenchym erheben. 


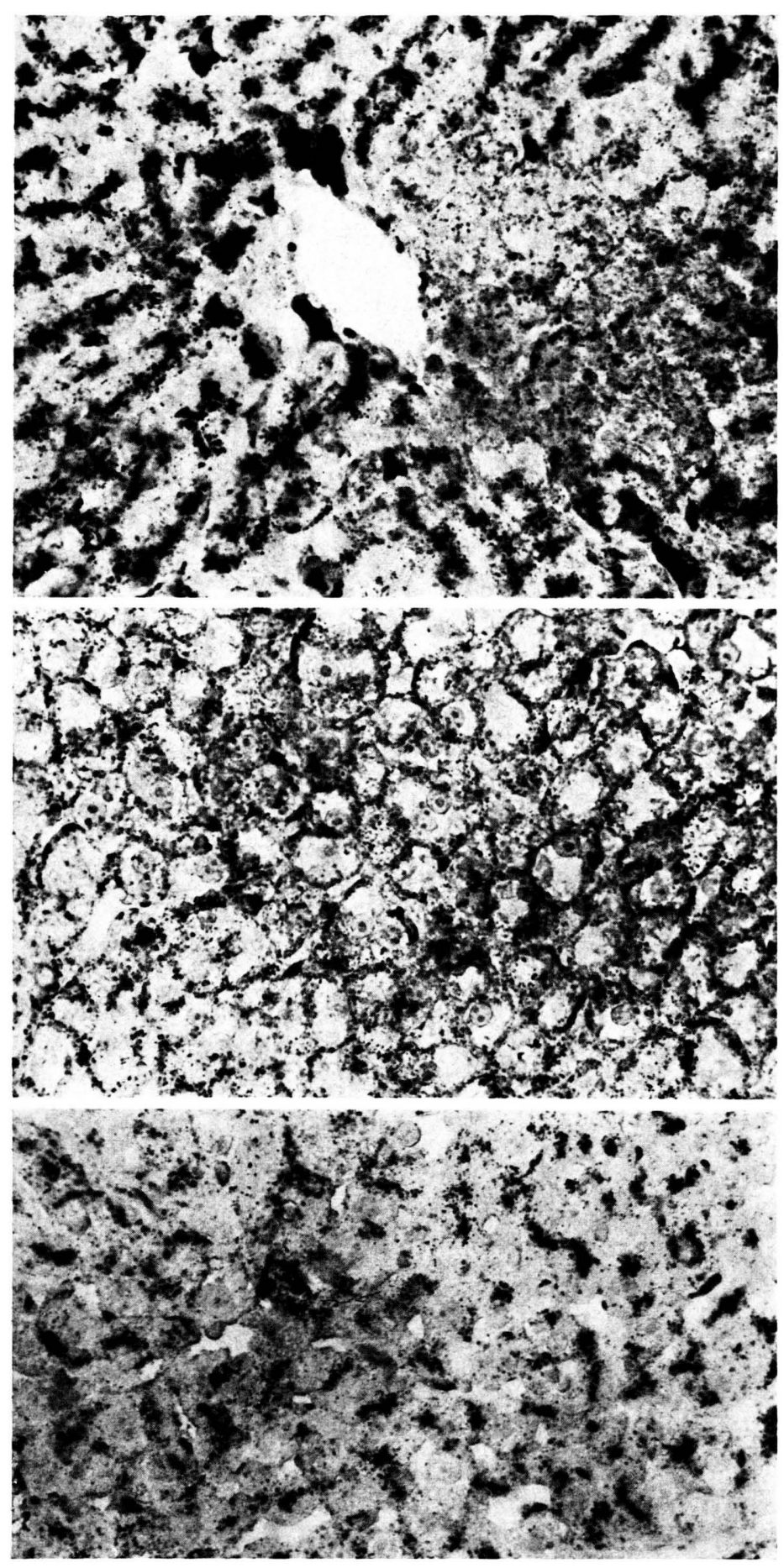

Abb. 3. Veränderungen der sauren Phosphatase unter dem Einfluß von DÄNA. a: Aufhebung der peribiliären Anordnung der enzymaktiven Granula, der Lysosomen, nach 7-wöchiger Fütterung von DÄNA. b: Verdrängung der Lysosomen an den Zellrand bei starker Glykogeneinlagerung in die Zelle. Durch Herauslösung des Glykogens erscheint das Zellinnere optisch leer. 10-wöchige Fütterung von DÄNA. c: Teilweise Wiederherstellung der peribiliären Anordnung der Lysosomen. 16-wöchige Fütterung von DÄNA. Vergr. 750-fach.

Abb. 3 a.

Abb. 3 b.

Abb. 3 c. 
Ein Verlust der ATPase-Aktivität in Gallenkapillaren der Rattenleber findet sich nach Verfütterung von Dimethylaminoazobenzol (Wachstein, Meisel und F ALCON ${ }^{11}$, nach toxischer Schädigung der Leber durch Thioacetamid $\left(\mathrm{W}_{\text {ACHSTEIN }}{ }^{12}\right)$, nach Infektionen mit Salmonella typhimurium (W $\mathbb{W}_{\text {ACHSTEIN, MEI- }}$

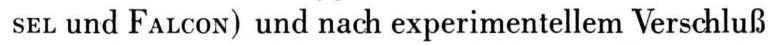
der Gallenwege (Novikoff und Essner) ${ }^{13}$. Gleiche ATPase-Verluste konnte Holzner ${ }^{14}$ auch in der menschlichen Leber bei toxischer Parenchymschädigung und bei Virusinfektionen nachweisen. Das Auftreten alkalischer Phosphatase in der Wand von Leberzellen wurde beobachtet nach Verabreichung der Cancerogene Ethionin und Dimethylaminoazobenzol sowie nach Infektionen mit Salmonella typhimurium (Wachstein und Meisel ${ }^{15}$; Wachstein, Meisel und Falcon). Diese enzymhistochemischen Veränderungen sind wohl der übereinstimmende Ausdruck einer gestörten Ausscheidungsfunktion der Leberzelle. Sowohl in der Leber wie in anderen Geweben charakterisiert eine hohe ATPase-Aktivität Membranen, die aktiv am Transport von Molekülen beteiligt sind. Aus dem Verlust der ATPase-Aktivität kann auf eine Störung eines Ausscheidungsmechanismus in diesen Wandabschnitten geschlossen werden (Essner u. a. ${ }^{16}$; Novikoff und Essner ${ }^{13}$ ). Dafür würde auch sprechen, daß zugleich die bevorzugte Anordnung der Lysosomen an diesen Wandabschnitten verschwindet. Auch das Auftreten alkalischer Phosphatase in der Wand von Leberzellen wird auf eine Störung bei der Ausscheidung dieses Enzyms aus der Zelle zurückgeführt ( $\left.\mathbb{W}_{\text {ACHSTEIN }}{ }^{17}\right)$.

Die bei Einwirkung von DÄNA auf das Leberparenchym sehr bald einsetzende Aufhebung der peribiliären Anordnung der Lysosomen, ihre Verminderung und diffuse Verteilung im Cytoplasma wurde auch bei anderen Alterationen des Leberparenchyms beobachtet, - nach toxischer Zellschädigung durch Thioacetamid und Tetrachlorkohlenstoff (Wachstein, Meisel und Falcon ${ }^{11}$ ), nach Verfütterung von Dimethylaminoazobenzol und nach Unterbindung der Gallenwege (Novikoff und Ess-

11 M. W 40, 219 [1962].

12 M. Wachstein, Gastroenterologia [Basel] 37, 525 [1959].

13 A. B. Novikoff and E. Essner, Amer. J. med. 29, 102 [1960].

14 J. H. Holzzer, Verh. dtsch. Ges. Path. 44, 233 [1960].

15 M. W Achstein and E. Meisel, J. Histochem. Cytochem. 7, 189 [1959].

16 E. Essner, A. B. Novikoff, and B. Masek, J. biophysic. biochem. Cytol. 4, 711 [1958].
NER ${ }^{13}$ ). Die diffuse Verteilung der Lysosomen im Cytoplasma und ihre Verminderung läßt daran denken, daß ein Teil derselben aufgelöst wird. Dadurch würde es zu einer Freisetzung hydrolytischer Enzyme im Zellinneren kommen. Ein Angriff an Cytoplasmabestandteilen wäre u. U. die Folge. Dies könnte die elektronenoptischen Beobachtungen einer teilweisen Auflösung des endoplasmatischen Reticulums in den frühen Phasen der Cancerogenese miterklären.

Die in einer späteren Phase der Cancerogenese bei starker Einlagerung von Glykogen im Cytoplasma beobachtete Zusammendrängung der Lysosomen an der Wand der Leberzelle findet ihre Parallele in den Befunden von Holzner u. a. ${ }^{18}$ bei grobtropfiger Verfettung der menschlichen Leberzelle. Auch dort ist das Cytoplasma weitgehend verdrängt. Lysosomen sind nur in einem schmalen Bereich entlang der Zellmembran nachweisbar.

Die Mitochondrien erfahren offenbar im Gegensatz zu anderen Zellorganellen - dem endoplasmatischen Reticulum, den Lysosomen und der Zellmembran - auch bei längerer intensiver Einwirkung von DÄNA auf die Leberzelle keine wesentlichen Veränderungen. Wir konnten lediglich eine Verminderung der Succinodehydrogenase-Aktivität beobachten. Eine ähnliche Herabsetzung der Aktivität dieses Mitochondrienenzyms fand sich auch nach Verabreichung des Cancerogens Dimethylaminostilben (Bitensky u. a. ${ }^{19}$ ). Ein völliger Verlust der Succinodehydrogenase tritt nur bei Nekrosen des Lebergewebes ein - z. B. infolge Thioacetamid oder Tetrachlorkohlenstoff (Leduc und Wilson ${ }^{20}$; Wachstein, Meisel und Falcon ${ }^{11}$ ).

Die bei unterschiedlichen Noxen übereinstimmend auftretenden enzymhistochemischen Veränderungen der ATPase, der alkalischen und sauren Phosphatase sowie der Mitochondrienenzyme können als ein empfindliches Maß für die Schwere einer die Leberzelle treffenden Schädigung angesehen werden. Eine umfassende Schädigung des Zellstoffwechsels wird nach rascher Störung der Enzymsynthesen durch das baldige Auftreten der oben beschriebenen enzym-

17 M. Wachstein, Cyto- and Histochemistry of the Liver, in : The Liver. Morphology, Biochemistry, Physiology I. Сн. Roulller, Edit., Academic Press, New York, London 1963. 18 J. H. Holzner, N. Stefenelli u. F. G. Wewalka, Wiener klin. Wschr. 74, 810 [1962].

19 L. Bitensky, R. W. Baldwin, and J. A. Chayen, Brit. J. Cancer 14, 690 [1960].

20 E. H. Leduc and J. W. Wilson, Arch. Pathol. 66, 147 [1958]. 
histochemischen Veränderungen manifest. Ein Beispiel dafür bieten die degenerativen Veränderungen der Leberzelle nach Verabreichung von Tetrachlorkohlenstoff, die in Stunden ablaufen (GössNer) ${ }^{21}$. Unter dem Einfluß cancerogener Dosen von DÄNA dagegen ist das Auftreten enzymhistochemischer Veränderungen über einen besonders langen Zeitraum auseinandergezogen. Die ersten Ausfälle der ATPase und sauren Phosphatase finden sich erst nach 7-wöchiger Fütterung von DÄNA. Eine Verminderung

21 W. Gössner, Referat auf dem 5. Internat. Kongreß für klinische Pathologie, Mexiko City Oktober 1963. der Succinodehydrogenase-Aktivität bleibt weitere Wochen aus. Alkalische Phosphatase tritt auch am Ende der Cancerogenese nur in der Wand einzelner Leberzellen auf. Offenbar führt DÄNA in cancerogenen Dosen nur langsam zu Veränderungen in der Synthese von Zellenzymen im Sinne einer partiellen Schädigung einzelner Funktionen und Strukturen der Leberzelle.

Anm. b. d. Korr.: Nach Fertigstellung des Manuskriptes erschien eine Mitteilung von A. Schauer (Verh. dtsch. Ges. Pathol. 50, 344 [1966]) über den histochemischen Nachweis verschiedener Nucleosid-5'-Tri-, Di- und Monophosphatasen in der Rattenleber während der Cancerisierung mit DÄNA.

\title{
Anticryptoccal Serum Factors in Experimental Liver Disease
}

\author{
Hans H. Gadebusch * \\ Medical Research Unit, Veterans Administration Hospital and the Department of Microbiology, \\ University of Michigan, Ann Arbor, Michigan \\ (Z. Naturforschg. 23 b, 1091-1095 [1968] ; eingegangen am 17. Januar 1968)
}

\begin{abstract}
Lysozyme and $\beta$-lysin activity in rat serum have been studied biochemically and microbiologically after induction of hepatic dysfunction. Under the conditions of these experiments $\beta$-lysin activity was adversely affected by liver necrosis or cirrhosis, while both factors ( $\beta$-lysin ** and lysozyme) were affected by hyperbilirubinemia. Lysozyme and $\beta$-lysin activity were unaffected in serum from rats afflicted with (a) malnutrition, (b) fatty liver in the absence of significant necrosis, (c) disturbance in porphyrin metabolism as represented by hexachlorobenzene intoxication, and (d) extent of glycogen deposition in the liver.

The potential role of these and other pertinent factors in host resistance to cryptoccosis has been discussed.
\end{abstract}

A recent study has shown ${ }^{1}$ that serum from certain patients with Hodgkins' granuloma, reticulum cell sarcoma, or leukemia exhibited anti-crytococcal activity which deviated from the normal. Exacerbation and subsequent restabilization of the neoplastic process could be charted by plotting the inhibitory activity of the serum. In addition, it was shown that some patients with hepatomegaly in the absence of obvious reticuloendothelial disease have yielded

* Present address: Squibb Institute for Medical Research, New Brunswick, N. J. 08903.

** The term $\beta$-lysin as commonly referred to in the literature today describes thermostable bactericidin (s) which is found in the serum of certain mammals and presumably has its origin in the blood platelet (J. G. Hirsch, J. exp. Medicine 112, 15 [1960] ; G. G. Hunder and R. F. JAcox, J. Immunology 90, 540 [1963] ; D. M. Donaldson, R. S. Jensen, B. M. Jensen, and A. Matheson, J. Bacteriol. 88, 1049 [1964]). While $\beta$-lysin like factors active against Cryptococcus neoformans $^{+}$have been reported as early as 1955 (W. P. ALLEN, Ph. D. Thesis - An Investigation of the anti-cryptococcal properties of normal serums. U. of Michigan, Ann Arbor, Mich.) their source and identity have remained unknown. Recently, H. IGEL and R. Bolande (. infect. Diseases 116, 75 [1966]), working with anti- $\beta$-lysin supplied by serums with reduced activity. It was thus the aim of the present study to define the role of the liver in decreased serum inhibition of crytococci by measuring this activity in rats with experimentally induced hepatic dysfunction.

\section{Materials and methods}

Animals - Male, Holtzman rats (Rowley Farms, Plymouth, Michigan) ranging in body weight from 160 to $180 \mathrm{gm}$., were used throughout this study. Each ex-

Donaldson, were unable to establish serological identity between serum anticryptococcal inhibitor and Donaldson's $\beta$-lysin. For sake of convenience the term $\beta$-lysin is used in this paper to indicate similarity to but not identity with the thermostable bactericidin.

+ Cryptococcus neoformans ist der Erreger der sogenannten Cryptococcose (Europäische Blastomykose Busse-Buschke, Torulose). Die Cryptococcose der Ratte wie beim Menschen nimmt ihren Ausgang von einer Inhalationsinfektion der Lungen. Metastasierung mit Befall von Nieren, Leber, Milz und Knochenmark ist häufig zu sehen. Öfters ist die Infektion bemerkbar bei Reticulosen, $\mathrm{H} \mathrm{o} \mathrm{d} \mathrm{g} \mathrm{k} \mathrm{i} \mathrm{n} \mathrm{scher-} \mathrm{oder}$ Zucker-Krankheit oder bei Krankheiten, wobei die Wirtsresistenz vermindert ist.

1 H. H. Gadebusch, Antimicrobial Agents and Chemotherapy - p. 640 [1964]. 